Revue d'histoire de l'enfance « irrégulière »

Le Temps de l'histoire

9 | 2007

Violences et jeunesse

\title{
Présentation du numéro : La violence, attribut et stigmate de la jeunesse
}

David Niget

\section{(2) OpenEdition \\ Journals}

Édition électronique

URL : http://journals.openedition.org/rhei/2653

DOI : 10.4000/rhei.2653

ISBN : 978-2-7535-1648-9

ISSN : 1777-540X

Éditeur

Presses universitaires de Rennes

Édition imprimée

Date de publication : 15 novembre 2007

Pagination : 9-21

ISSN : 1287-2431

Référence électronique

David Niget, « Présentation du numéro : La violence, attribut et stigmate de la jeunesse », Revue d'histoire de l'enfance « irrégulière » [En ligne], 9 | 2007, mis en ligne le 02 novembre 2009, consulté le 04 décembre 2020. URL : http://journals.openedition.org/rhei/2653 ; DOI : https://doi.org/10.4000/rhei. 2653 


\section{La violence, attribut et stigmate de la jeunesse}

Aborder la question de la jeunesse dans son rapport à la violence, pour le chercheur novice comme pour l'universitaire confirmé, exige à la fois largeur de vue et densité de l'analyse. ${ }^{(2)}$ Car, si cet intitulé à la tournure " anthropologique " paraît vaste, voire sans fond, il requiert de celui qui s'y risque une solide réflexion, dans la mesure où le paradigme de violence, par sa polysémie même, appelle une lecture orientée, une interprétation des phénomènes historiques, loin des béates observations positivistes. Traiter de violence consiste, pour le chercheur, à multiplier les points de vue : celui du perpétrateur, celui de la victime, mais encore celui des témoins directs et indirects, pour accéder au regard social posé sur le geste, avant, finalement, de considérer sa propre perspective et celle "de son temps", selon une démarche réflexive. Car ce n'est rien de compter les coups, d'ausculter les blessures, de rapporter les faits d'arme, de sentir sourdre la vengeance, si l'on n'est pas en mesure d'inscrire ces actes au-delà de la sidération qu'engendre la brutalité. Il ne s'agit pas de choisir entre une approche culturaliste de la violence, et une autre qui soit matérialiste, mais plutôt de miser sur un réalisme constructiviste, qui reconnaisse à la fois la rudesse des coups et la voie spécifique selon laquelle ils affectent l'ethos des acteurs, et sont médiatisés dans l'espace social.(3)

Évacuant d'emblée toute neutralité - imaginaire - du chercheur, la question de la violence exercée et subie par la jeunesse se révèle être, bien plus qu'un "sujet d'actualité ", un véritable fil épistémologique, mobilisant toutes les ressources des sciences sociales en plus de la spécificité de la discipline historique, comme le souligne Xavier Rousseaux en conclusion de ce volume. Car la violence n'est pas indifférente à la dimension diachronique des phénomènes sociaux. Elle relève d'un processus, qui tantôt arme son bras destructeur, tantôt permet son acclimatation au jeu social sous la forme du
David Niget $^{(1)}$

(1) Chercheur post-doc-

toral au sein de l'unité

Droit et changement

social, de l'université de

Nantes (CNRS, UMR

6028), et du Centre

d'histoire du droit et de la

justice de l'université

catholique de Louvain.

Je remercie

Xavier Rousseaux pour

ses suggestions.

(2) Les travaux réunis

dans ce dossier sont issus

d'un séminaire doctoral

tenu en 2005 à l'université

catholique de Louvain

sous la direction de Xavier

Rousseaux et de David

Niget. Je tiens à remercier

tous les participants

d'avoir enrichi de leurs

interventions cette

réflexion collective : en

plus des contributeurs à ce

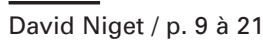


volume de la revue RHEI,

Mmes Fabienne Brion,

Aurore François, Salima

El Karouni, Karolien

Declerq, Françoise Muller,

Pascale Quincy-Lefebvre,

Sylvette Dupont-

Bouchat ; MM. David

Somer, Pierre Bar,

Jean-Marie Fecteau,

Jean-Jacques Yvorel,

Jean-Claude Caron et

Jean-Noël Luc.

(3) Jean-Claude Farcy appelait de ses vœux ce type d'approche de la violence dans un bilan historiographique consacré à l'histoire de la justice. Jean-Claude Farcy, L'histoire de la justice française de la Révolution à nos jours. Trois décennies de recherches, Paris, PUF, 2000, p. 123-125.

(4) Georg Simmel, Le conflit, Paris, Circé, 1995, 163 p. (Initialement publié dans : Sociologie. Études sur les formes de la socialisation, textes publiés entre 1894 et 1917.$)$ conflit fécond, comme l'a montré Georg Simmel.(4) Ainsi, la violence est à réinscrire dans le cycle beaucoup plus vaste des luttes des individus et des groupes sociaux pour "faire société ". Elle renvoie également au changement social, à "l'horizon d'attente » des acteurs sociaux, tant il est clair que les frustrations engendrées par la perception d'un avenir obscur en constituent la source, spécialement s'agissant de la jeunesse. ${ }^{(5)}$

Comment concevoir, sans diluer la problématique, le croisement entre deux objets, jeunesse et violence, aux contours si changeants à travers le temps, que l'on se penche sur la vulgate des pratiques sociales ou que l'on prête attention aux discours savants et médiatiques ? Car le sujet est captieux. Selon les canons de la culture occidentale, chrétienne, leur juxtaposition sonne comme un oxymoron. La jeunesse, et plus encore l'enfance, incarnent le temps de l'innocence. Leur association relève d'emblée de l'anomalie, qui, de la casuistique morale jusqu'à la psychiatrie clinique, sera mise en exergue. A contrario, le sens commun, redoublé par la culture savante, insiste sur la culture belliqueuse de la jeunesse, ce que viennent corroborer les statistiques pénales au XIXème siècle. Ainsi, Adolphe Quételet, en 1831, s'alerte de la disposition criminelle de la jeunesse, associant le crime à une sauvagerie juvénile conditionnée par un corps livré à ses désirs :

"Lâge est sans contredit la cause qui agit avec le plus d'énergie pour développer ou pour amortir le penchant au crime. Ce funeste penchant semble se développer en raison de l'intensité de la force physique et des passions de l'homme ; il atteint son maximum vers l'âge de 25 ans, époque où le développement physique est à peu près terminé. Le développement intellectuel et moral, qui s’opère avec plus de lenteur, amortit ensuite le penchant au crime qui diminue encore plus tard par l'affaiblissement de la force physique et des passions de l'homme. [...] l'homme, poussé par la violence de ses passions, se livre d'abord au viol et aux attentats à la pudeur ; il entre presque en même temps dans la carrière du vol qu'il semble suivre comme par instinct jusqu’à son dernier soupir ; le développement de ses forces le porte ensuite à tous les actes de violence, à l'homicide, à la rébellion, aux vols sur les chemins publics ; plus tard, la réflexion convertit le meurtre en assassinat et en empoisonnement. Enfin, l'homme en avançant dans la carrière du crime, substitue de plus en plus la ruse à la force, et devient faussaire jusqu’à toute autre époque de sa vie. " ${ }^{\left({ }^{(}\right)}$ 
En outre, sur le plan méthodologique, les deux catégories de jeunesse et de violence partagent la caractéristique de s'imposer d'elles-mêmes comme données, universelles, sans histoire. Quoi de plus « naturel », d'une part, qu'un " âge de la vie " ? D'autre part, tout individu ne fonde-t-il pas dans son expérience sociale une connaissance intime de la violence, marquée dans les corps et les consciences par la souffrance ou la peur ? (7) Et pourtant, sous l'œil de l'ontologiste, violence ou jeunesse s'avèrent n'exister finalement que relativement à leur environnement, comme relation plus que comme substance, comme phénomène construit plus que comme réalité objective. Ainsi, il n'est de violence que dans la corrélation entre une force exercée ou subie, et le dépassement d'un seuil de tolérance à cette force, dépassement qui qualifie cette force comme de la violence. De même, il n'est de jeunesse que dans le lien entre ce stade biologique, et la position relative de ce groupe par rapport à d'autres classes d'âges elles-mêmes socialement construites, préexistantes, et à ce titre, disposant de la capacité de désigner le groupe "jeunesse ». Entre naturalisme et relativisme, quelle posture peut adopter le chercheur? Et quels fils tirer pour dévoiler cet objet double offrant peu d'aspérités ?

On le voit, derrière ce piège de la doxa sont tapis des enjeux spécifiques, liés à la fabrique des catégories sociales, et au langage pour les désigner. Définir devient à leur égard une tentative de fixation, qui admette cependant ne pas être un exercice fini, car ces objets sont en perpétuelle reconfiguration. En outre, les analyser de manière diachronique, processuelle, dans la longue durée qui fait la spécificité de la discipline historique, permet de se départir de cette tentation de la naturalisation, en observant les conditions changeantes de configuration de ces catégories. Il s'agit bien ici de proposer une socio-histoire des violences exercées ou subies par la jeunesse, à travers le prisme de plusieurs études de cas, balayant de concert plus de cinq siècles d'histoire, articulant observation locale et nationale, voire transnationale, puisque Belgique et France ont emprunté des modèles et échangé des expériences au long des deux derniers siècles, tout en ayant été confrontées à des configurations socio-politiques très différentes (pensons, par exemple, à l'expérience d'occupation qui frappe la Belgique dès la première guerre). ${ }^{(8)}$

Ce recueil se présente comme une invitation à de plus amples recherches. ${ }^{(9)}$ Il signale en creux la nécessité d'une analyse comparatiste, dans la longue

(5) Reinhart Koselleck, Le futur du passé. Contribution à la sémantique des temps historiques, Paris, Éditions de l’École des hautes études en sciences sociales, 1990, 334 p.

(6) Adolphe Quételet, "Recherches sur le penchant au crime aux différents âges ", Nouveaux mémoires de l'Académie des sciences et belles-lettres de Bruxelles, t. VII, Bruxelles, Hayez, 1831, p. 74-75.

(7) Joanna Bourke, Fear. A Cultural History, London, Virago, 2005, p. 330-331.

(8) Si l'on connaît relativement bien l'histoire de la violence interpersonnelle du Moyen Âge à la première guerre mondiale, un chantier s'ouvre maintenant pour les historiens du XXème siècle, en Belgique comme en France. Ginette Kurgan- 
van Hentenryk, [dir.],

Un pays si tranquille:

la violence en Belgique au

XIXème siècle, Bruxelles,

Éditions de l'université de

Bruxelles, 1999, 253 p. ;

Frédéric Chauvaud,

De Pierre Rivière à

Landru : la violence apprivoisée au XIXème siècle,

Paris, Brépols,

1991, 271 p.

(9) Signalons le démarrage, en octobre 2007,

à l'université catholique de Louvain, d'une recherche pluridisciplinaire de cinq années sur le thème :

" Jeunesse et violence en Belgique, 1880-2006 : approches socio-historiques ». Cette équipe de jeunes chercheurs sera dirigée par Mme Fabienne Brion, directrice de l'École de criminologie, et MM. Jean-Michel Chaumont, professeur de sociologie, et Xavier Rousseaux, professeur d'histoire. durée et au sein de plusieurs espaces souverains ou nationaux, qui dépasse les apories inhérentes à toute tentative de compréhension d'un phénomène enferré dans des contingences spécifiques mais le plus souvent invisibles, car dissoutes dans les structures et représentations sociales d'une communauté singulière. Tel est le cas des « seuils de tolérance " à la violence, souvent invoqués, mais difficilement mensurables. Par ailleurs, les études de longue durée et transnationales consacrées à l'homicide ont montré que l'on pouvait apporter des réponses transversales à un objet - le meurtre -, qui paraissait, aux yeux des historiens, irréductible à une analyse structurale. ${ }^{(10)}$ En contrepoint des approches comparatives, les études de cas ici réunies invoquent la nécessité d'observer la construction socio-culturelle de la violence juvénile à travers la densité du microcosme qui l'a produite (la famille, le village, le quartier, l'institution de correction, la nation...). Il s'agit, en marchant dans les pas de Clifford Geertz, de saturer le contexte observé et d'accéder, derrière les faits, à la signification des situations paroxystiques qu'engendre la violence. ${ }^{(11)}$ On peut ainsi espérer réinscrire l'accidentel dans un horizon de sens, celui des acteurs eux-mêmes, et celui de la société qui l'a engendré. Une série de questions générales, consignées dans des études particulières, jalonnent le parcours proposé par les auteurs de ce volume.

\section{Jeunesse et violence : pour une critique de la genèse sociale des catégories}

Il s'agit d'abord d'envisager comment, plutôt que de relever d'un lien « naturel », le processus d'assignation de la violence à la catégorie des jeunes est socialement construit. Comme valeur constitutive de leur identité sociale, la violence octroie aux jeunes certaines prérogatives - à condition d'en faire un usage mesuré à des fins spécifiques -, mais elle les confine en retour dans un espace politique restreint, car, in fine, seule la sagesse confere le pouvoir. ${ }^{(12)}$ Les frontières de la jeunesse sont ainsi l'objet de luttes de classement, où l'âge biologique s'articule de manière complexe avec l'âge social. Ne peut-on pas, nous expliquent Aude Musin et Élise Mertens, être considéré comme " jeune » à 45 ans sonnés, au Moyen Âge, pour peu que l'on soit célibataire, ou dépendant de ses parents ? À distance historique, ces auteures signalent que jeunesse et violence sont deux objets mouvants aux XIVème et XVème siècles : 
leur définition est éminemment variable et leur association ne va pas de soi. La justice, qui identifie de plus en plus fréquemment la jeunesse des accusés et de ses requérants en grâce, assimile tantôt la jeunesse à une excuse, tantôt à un péril. Tout au long des Temps modernes, plus que le jeune, c'est le vagabond qui cristallise les peurs d'une société, alors que la mobilité des travailleurs est entravée par le poids des corporations et que les garanties sociales sont octroyées par la communauté locale. ${ }^{(13)}$ Les jeunes en situation précaire qui se lancent sur les routes deviennent alors des populations " à risque ", cumulant deux stigmates.

Ainsi, du Moyen Âge au XIXème siècle, la jeunesse violente n'est a priori pas condamnée pour ses agissements, pour autant qu'elle défende les coutumes et les identités communautaires. Comme le montre François Ploux, les jeunes sont les gardiens de l'ordre à l'intérieur (charivaris) comme à l'extérieur ("guerres" paysannes) de la communauté villageoise du XIXème siècle. En outre, la violence, intimement liée aux mécanismes de reproduction sociale, est le lot d'une jeunesse qui se place ainsi sur le marché matrimonial. Aussi, dans la longue durée, la violence n'apparaît pas seulement comme un stigmate, mais comme un attribut de la jeunesse. Malgré la domestication de la violence juvénile qui caractérise le XXème siècle, ce trait identitaire n’a pas disparu aujourd'hui. Cette expressivité vindicative est en droit d'être entendue, reconnue (et à ce titre encadrée), mais nous assistons cependant à un processus de disqualification d'une partie de la jeunesse, accusée de violence " gratuite ". Ce stigmate signale, dans le discours des élites, non seulement sa dangerosité mais surtout son incapacité à prendre part à la communauté politique, ce qui justifie en retour sa mise à l'écart.

\section{Violence exercée, violence subie :}

\section{du risque criminel à la protection de la jeunesse}

Comme le rappellent la plupart des auteurs, la violence est simultanément exercée par les jeunes et subie par eux. D'abord, les premières victimes des groupes de jeunes sont d'autres jeunes. En effet, l'agression se dirige avant tout vers l'alter ego, le concurrent dont il est difficile de se distinguer, et selon une intensité plus forte encore que la proximité sociale est grande, comme le rappelle François Ploux. En outre, la violence, familière à l'enfant qui en est fré-
(10) Manuel Eisner,

«Modernization, Self-

control and Lethal

Violence. The Long-term

Dynamics of European

Homicide Rates in

Theoretical Perspective ",

British Journal of Crimi-

nology, 41, 4, Autumn

2001, p. 618-638.

(11) Clifford Geertz,

"La description dense.

Vers une théorie interprétative de la culture ",

Enquête, 6, 1998,

p. $73-105$.

(12) Pierre Bourdieu, "La “jeunesse” n'est qu'un mot ", Questions de sociologie, Paris, Éditions de Minuit, 1984, p. 143-154.

(13) Robert Castel, Les métamorphoses de la question sociale. Une chronique du salariat, Paris, Fayard, 1995, en particulier le chap. III : "Indigne salariat». 
(14) Dominique Kalifa, " "Dangerosité” et "défense sociale" au début du XXème siècle ", Crime et culture au XIXème siècle, Paris, Perrin, 2005, p. $257-270$.

(15) Voir les articles de Dominique Dessertine, Jean-Jacques Yvorel, Geoffroy Le Clercq, etc., in le Temps de l'histoire, $\mathrm{n}^{\circ} 2$, "Cent ans de répression des violences à enfants ", mai 1999, $221 \mathrm{p}$.

(16) Notons que Weber a recours au mot de gewalt, qui signifie autant force que violence. Max Weber, Le savant et le politique, Paris, La découverte, 2003 (1919), p. 118.

(17) Voir aussi JeanClaude Farcy, « Jeunesse rurale et rixes villageoises au XIXème siècle ", in Jean-Claude Vimont [dir.], Jeunes, déviances et identités, XVIIIème-XXème quemment témoin ou victime dans le cadre familial, ressurgit lorsqu'il atteint l'âge de l'exercer, processus bien connu des psychologues aujourd'hui. Enfin, la brutalité est inhérente à la construction sociale des rapports de sexes, qui voient les violences sexuelles être fréquemment exercées par des jeunes hommes sur des adolescent(e)s ou des enfants.

À la dichotomie simpliste de l'enfant victime ou coupable, on peut opposer des situations et des trajectoires de jeunes qui sont à la fois " en danger " et " dangereux ». Ainsi, la notion de risque devient centrale à la fin du XIXème siècle, qu'il s'agisse du risque encouru par le mineur ou de celui qu'il fait courir à la société. La Belgique, berceau de la doctrine de la défense sociale, donne un vivant exemple de cette tension fondatrice entre protection de l'enfance et protection de la société. ${ }^{(14)}$ En France, les lois de 1889 sur la déchéance paternelle et de 1898 sur les violences à enfants procèdent de cette présomption que les jeunes victimes sont le terreau de la délinquance à venir. ${ }^{(15)}$

Plus encore, les victimes - incarnées dans ce volume par les vagabonds, les filles violées ou les gamins de l'Assistance -, sont tout aussi dangereuses que les coupables dans l'esprit des observateurs et intervenants, voire plus, car elles n'ont rien à perdre, et peuvent puiser dans leur désespoir la rage attisant une vengeance irréfléchie et donc incontrôlable. Ivan Jablonka retrace avec sagacité la généalogie de la violence « sadique " que les pupilles de l'Assistance exercent sur les bêtes, retournant ainsi la violence symbolique ou même physique qu'ils ont eux-mêmes subie. Maurice Pialat avait noté aussi cet indice de la souffrance du jeune déraciné, quand, dans L'enfance nue, le jeune François martyrise le chat de sa famille d'accueil en le jetant dans la cage d'escalier sous le regard sidéré et admiratif - de ses jeunes voisins. Au massacre du chat succède une scène touchante où le jeune garçon soigne, seul, l'animal brisé, indice qu’à la cruauté se superpose aussi la fraternisation bienveillante avec des animaux au triste sort, compagnons d'infortune des enfants perdus.

Ainsi, la violence s'inscrit dans la circulation sociale des affects plus que sous la forme - souvent fantasmée - du surgissement individuel.

\section{La violence entre l'individu, le corps social et l'État}

La violence ne renvoie pas seulement à l'individu. Comme nous le rappellent les auteurs, elle couve dans le corps social. Surtout, la construction du 
phénomène est indissociable de l'édification de l'État moderne, selon la thèse centrale de Max Weber, qui a identifié ce dernier comme le vecteur par lequel une élite sociale réussissait à imposer le monopole de l'exercice de la violence légitime. ${ }^{(16)}$ Le moins que l'on puisse dire, à lire Aude Musin et Élise Mertens, puis François Ploux et Aurélien Lignereux pour le XIXème siècle, c'est que ce monopole est contesté ! Les communautés sont désireuses de conserver leurs guerres privées, rythmées par le cycle des vengeances et la défense du point d'honneur. Bien sûr, l'intensité de ce conflit n'est pas la même au XVème siècle, alors que l'État est embryonnaire, et au XIXème, alors qu'il se superpose définitivement aux pouvoirs locaux. La confrontation avec les gendarmes témoigne alors du bras de fer qui s'instaure entre un État nation en construction et des campagnes au faîte de leur puissance démographique, force vitale incarnée par la jeunesse.

Le dernier tiers du XIXème siècle est marqué par le recul des violences juvéniles collectives ritualisées, qui avaient pourtant été une pierre de touche de l'organisation des sociétés traditionnelles, comme le démontre François Ploux. ${ }^{(17)}$ C'est la fin des charivaris, carnavals et guerres paysannes propres au milieu rural, dans lesquelles les groupes de jeunesse occupaient une position centrale comme défenseurs des intérêts de la communauté. ${ }^{(18)}$ Le lent processus de " civilisation des mœurs ", décrit par Norbert Élias, agit désormais, par l'intermédiaire des élites locales, et sous l'aiguillon d'un État de plus en plus puissant, au sein de classes populaires intériorisant le contrôle des affects comme élément constitutif de la socialité. ${ }^{(19)}$

Grâce à l'article de Jérôme de Brouwer consacré à l'application de la peine capitale aux mineurs, on perçoit bien ce tiraillement, au croisement des analyses weberienne et eliassienne. D’une part, l'État, selon l'idéologie libérale " progressiste ", doit se faire pédagogue de la pacification des mœurs en administrant les peines avec retenue afin d'édifier une société de progrès, et d'autre part, il se doit d'étêter - au sens propre comme au figuré ! - toute velléité parricidaire, figure suprême de la menace politique, selon un discours conservateur assimilant le meurtre du père à celui du roi.

Dans ce siècle positiviste, la violence, de phénomène social reconnu, devient un attribut individuel pathologique, que les sciences du psychisme et la criminologie triomphantes vont tendre à stigmatiser. Marqueur de l'adolescence, la siècles, Cahiers du GRHis, $\mathrm{n}^{\circ} 15$, Mont-Saint-

Aignan, Publications des universités de Rouen et du Havre, 2005, p. 23-34.

(18) Malgré la disparition du charivari, il est intéressant de signaler, avec Xavier Rousseaux (dans ce volume), les résurgences de violences juvéniles ritualisées au XXème siècle, alors que le pouvoir légitime entre en crise, notamment lors des entrées et sorties de guerre.

(19) Pour Élias, qui s'oppose à l'analyse de Weber, « il y a interdépendance étroite entre structures sociales et structures émotionnelles. Il n'existe pas de puissance coercitive capable d'imposer aux hommes la modération. Quand dans telle ou telle région, le pouvoir central s'affermit, quand il oblige les hommes sur un territoire plus ou moins étendu de 
vivre en paix, on assiste aussi à un changement progressif de l'affectivité et des normes de l'économie pulsionnelle ». Norbert Élias, La civilisation des mours, Paris, CalmanLévy, 1991 (1939), p. 292-293.

(20) L'étude de Sylvie Lapalus confirme en effet que le parricide est avant tout affaire de transmission patrimoniale. La mort du vieux. Une histoire du parricide au XIXème siècle, Paris, Tallandier, 2004, 633 p.

(21) David Niget, "Venal Sexuality, Predatory Sexuality, or Pathological Sexuality? Girls and Boys

Confronting the Juvenile Justice System in Angers (France), 1914-1945 ", in Jean Trépanier, Éric Pierre, Xavier Rousseaux, [dir.], Juvenile Crime and Justice in the XXth Century, [à paraître].
(22) Walter Benjamin, "Critique de la violence ", Euvres, t. I, Paris, Gallimard, coll. « Folio Essais ", 2000 (1921), p. $210-243$.

(23) Jean-Clément Martin, Violence et Révolution française : essai sur la naissance d'un mythe national, Paris, Le Seuil, coll. « L'univers historique », 2006, 338 p.

(24) Michelle Perrot,

Les ouvriers en grève :

France, I87I-I89o, Paris-

La Haye, Mouton, 1973, vol I : p. 313-318,

"Grèves de jeunes "; vol. II, p. 458-460.

(25) Georges L. Mosse, De la Grande Guerre au totalitarisme. La brutalisation des sociétés européennes, Paris, Hachette, 1999, spécialement le chap. III : « Les jeunes et la guerre ", p. 65-82. violence est alors le signe d'une double frustration sociale et sexuelle. Antichambre d'une maturité associée à l'autonomie économique, la jeunesse est le temps social de la convoitise et de l'impatience, sentiments qui peuvent mener au crime, ce que l'importance des jeunes parricides dans l'imaginaire collectif vient confirmer. ${ }^{(20)}$ C'est aussi la montée de l'instinct sexuel qui caractérise l'adolescence, qui fait du jeune livré aux injonctions de ses sens un prédateur ou une nymphomane, tous deux considérés comme un danger social. ${ }^{(21)}$

Le lent processus de pacification de la société, dont la disciplinarisation de la jeunesse est un vecteur privilégié, ne doit pas occulter l'usage monopolistique de cette violence par le pouvoir légal, comme le soulignaient Max Weber - on l'a dit - ou encore Walter Benjamin. ${ }^{(22)}$ Fondatrice de tout ordre social - que l'on pense aux révolutions démocratiques -, la violence organise et justifie les dominations qui caractérisent les sociétés, y compris lorsqu'elles se revendiquent du libéralisme. ${ }^{(23)}$ A contrario, la révolte brutale reste un moyen d'action privilégié pour les classes dominées pour se faire entendre dans le jeu social. Ainsi, les sociétés démocratiques, qui entendent à la fois pacifier les rapports sociaux et ouvrir un espace régulé à la conflictuosité comme gage de pluralisme, oscillent donc entre volonté d'éradiquer la violence et son instrumentalisation, en ouvrant des interstices de violence régulée, comme la grève légale, à laquelle la jeunesse va prendre une part importante, de la " jeunesse de la grève " aux manifestations estudiantines du second XXème siècle. ${ }^{(24)}$

La philanthropie du XIXème siècle avait pensé la fin de violence, voyant dans l'enfance et la jeunesse un terrain privilégié d'acculturation. Le premier XXème siècle 
bascule vers la «brutalisation de sociétés européennes ». La jeunesse devient alors un viatique pour le renouvellement des cultures politiques, à travers les organisations de jeunesse notamment. L'autoritarisme politique, grande tentation de l'Entre-deux-guerres, promeut alors l'exercice de la violence tout en l'encadrant à l'extrême. (25) L'étude des pratiques sociales nuancent fortement cette analyse culturelle, mais il est indéniable que la culture politique profere alors une injonction contradictoire, entre fascination pour une violence régénératrice et poursuite de la civilisation des mœurs. Le providentialisme postérieur à 1945 relève de nouveau de l'utopie historiciste d'une société pacifiée, aboutie. Selon un processus ambigu, se dessine un projet d'enserrement et d'éradication de la violence, que décrit bien Anne Thomazeau à travers les institutions pour filles délinquantes. "Déconditionnement ", " reconditionnement ", " personnalisation " deviennent les rouages d'institutions totales, selon le mot d'Erving Goffman, usant autant de techniques disciplinaires coercitives - parmi lesquelles les neuroleptiques font une apparition notoire - que de la responsabilisation individuelle, pour produire des individus pacifiques, autonomes, et socialement productifs. ${ }^{(26)}$

Ainsi, si la violence juvénile comme pratique sociale reconnue est peu à peu évincée par le processus de civilisation, cette évolution n’est ni linéaire, ni univoque. La violence reste au cœur des relations sociales, enchâssée dans un système politique qui l'a accaparée et domestiquée pour en faire un outil de gouvernement de la jeunesse.

\section{Le genre de la violence : une affaire d'hommes (jeunes) ?}

Les concepts de jeunesse et de violence révèlent une autre symétrie, issue tant des représentations communes que de l'approche scientifique : ils résistent au critère du genre ou mieux, ils l'occultent. Comme le souligne Michelle Perrot, la jeunesse a souvent été pensée au masculin. ${ }^{(27)}$ Tant à travers l'édification de la notion d'adolescence au début du siècle, chez le psychologue américain Stanley-Hall, ${ }^{(28)}$ que dans les études sociologiques consacrées à la jeunesse dans les années 1930-1960, (29) c'est le jeune garçon ou le jeune homme qui incarne la jeunesse. Tout se passe comme si la masculinité était déterminée par le statut de jeunesse, alors que la jeunesse était indifférente ou absorbée par le fait d'être femme. Les études féministes ont, depuis trente ans, compensé cette myopie,
(26) Voir aussi Abigail Wills, « Delinquency,

Masculinity and Citizenship in England, 1950-1970 ", Past and Present, 187, May 2005, p. 157-186.

(27) Michelle Perrot, "La jeunesse ouvrière, de l'atelier à l'usine ", Giovani Levi, Jean-Claude Schmitt, [dir.], L'histoire des jeunes en Occident, t. 1, Paris, Le Seuil, 1996, p. 122.

(28) Granville StanleyHall, Adolescence, its Psychology and its Relation to Physiology, Anthropology, Sociology, Sex, Crime, Religion and Education, New York, D. Appleton, 1904, 2 vol.

(29) Clifford R. Shaw, The Jack-Roller : A Delinquent Boy's Own Story, Chicago, Chicago University Press, 1930, 205 p. ; Norbert Élias, John L. Scotson, Logiques de l'exclusion. Enquête 
sociologique au cour des problèmes d'une communauté, Paris, Fayard, 1997, 278 p. (Trad. de The Established and the Outsiders, Sage, 1965.)

(30) Agnès Thiercé,

«De l'école au ménage : le temps de l'adolescence

féminine dans les milieux populaires (IIIÌme

République) ", Clio, $\mathrm{n}^{\circ} 4$,

«Le temps des jeunes

filles ", 1996 ; Whitney

Walton, «American Girls and French Jeunes Filles :

Negotiating National

Identities in Interwar

France ", Gender and

History, 17, 2, August

2005, p. 325-353. Deux

études fondamentales :

Thomas W. Laqueur,

La fabrique du sexe. Essai

sur le corps et le genre en

Occident, Paris, Gallimard,

1992, 355 p. (1ère éd. :

1990) ; Judith Butler,

Trouble dans le genre.

Pour un féminisme de la

subversion, Paris,

La découverte, 2005,

283 p. (1ère éd. : 1990).
(31) Voir les travaux

d'Arlette Farge consacrés au XVIIIÌme siècle pari-

sien, notamment Effusion

et tourment, le récit des

corps: Histoire du peuple au XVIIIème siècle, Paris,

Odile Jacob, 2007, 248 p. ;

et encore Cécile Dauphin,

Arlette Farge, Luc

Capdevilla, Jean-Clément

Martin, Rose-Marie

Lagrave, Françoise

Héritier, « Femmes et vio-

lence ", Le mouvement

social, 189 , octobre-

décembre 1999,

p. $83-110$.

(32) Georges L. Mosse, The Image of Man :

The Creation of Modern

Masculinity, Oxford,

Oxford UP, 1998, 240 p. ;

Clive Emsley, Hard Men :

The English and Violence

Since 1750, London,

Hambledon \& London, 2005, 288 p.

(33) Julia Grant, «A

Real Boy and not a Sissy : Gender, Childhood, and Masculinity, 1890- travaillant sur la distinction entre le sexe biologique et le genre social, la construction de la féminité et, plus récemment, de la masculinité, notamment à l'adolescence. ${ }^{(30)}$

De la même manière, la violence juvénile a le plus souvent été attribuée aux jeunes mâles. Aude Musin et Élise Mertens de Wilmars évoquent une société de violence principalement masculine, où la prévalence du viol parmi les jeunes garçons fonctionne de pair avec une tolérance judiciaire à l'égard des violences suscitées par la défense physique des filles du village menacées par des prédateurs étrangers. Si cette construction socio-politique n'a pas prévalu en toutes circonstances, voyant les femmes recourir publiquement à la violence en milieu urbain notamment, ${ }^{(31)}$ les XIXème et XXème siècles ont octroyé l'apanage de la violence aux hommes, associant même son exercice avec les valeurs culturelles de la masculinité, réifiée en "virilité ". C'est ce qu'illustre parfaitement François Ploux, qui voit dans les " guerres villageoises " le théâtre de l'expression identitaire des jeunes garçons, à travers l'exercice du combat. ${ }^{(32)}$ Plus près de nous, les experts de l'enfance ont considéré, au début du XXème siècle, qu'il s'agissait d'une part nécessaire de leur identité masculine, repoussant ainsi le spectre de la "féminisation " des sociétés occidentales, source de faiblesse, d'amoralisme et de consumérisme. ${ }^{(33)}$

Derrière ce privilège apparent de l'exercice de la violence, des études novatrices montrent bien que plusieurs procédés sont à l'œuvre pour déformer et occulter cette violence féminine dans l'espace social. D'une part, acteurs judiciaires et médias ont tendance à " masculiniser " les auteures de violences, avec la figure de la virago, garçonne dénaturée. ${ }^{(34)} \mathrm{D}$ 'autre part, malgré cette sorte de monstruosité de la violence féminine, elle fait l'objet d'un traitement judiciaire plus indulgent que 
lorsqu' elle est exercée par les " mauvais garçons ", car les filles sont considérées comme plus impressionnables et moins dangereuses. Cependant, il existe, comme le montre Anne Thomazeau, un décalage symptomatique entre la faiblesse des incriminations pour faits de violence chez les filles envoyées en institutions de rééducation, et le fait que cette violence soit un fait " attendu » de la part des jeunes filles internées. Attendu ne signifie en rien reconnu comme légitime, puisque la révolte féminine, qu'elle soit individuelle ou collective, est le plus souvent traitée sous l'angle de la déficience mentale, réveillant la figure de l'hystérique, selon laquelle la violence féminine est directement imputée à sa physiologie sexuelle (on parle au XVIIIème siècle de "fureur utérine "), puis à une maladie nerveuse spécifiquement féminine. ${ }^{(35)}$

Ce traitement de la violence féminine relève du déni de reconnaissance des jeunes filles dans l'espace de la Cité, si ce n'est comme victime d'agressions sexuelles. Le corps de la jeune fille n'apparaît comme menaçant que lorsqu'il est livré à la dépravation, et à ce titre, la violence qui lui est faite devient une violence faite à la société, d'où la confusion des registres de la culpabilité et de la victimation. ${ }^{(36)}$ Même abusée sexuellement, la jeune fille est fautive.

La violence féminine relève de la dénaturation, ce qui en fait une monstruosité mais en occulte en retour la visibilité sociale et apaise sa répression. Lorsqu'elle est dénoncée, cette violence est fondée sur la matrice sexuelle de l'agressivité, confondant alors les positions de victime et d'agresseur, de sujet et de malade. Ici encore, la jeune fille est rapportée à son sexe, et ainsi privée de ses droits.

\section{Entre rage et révolte : anomie ou horizon de sens ?}

Quant à l'interprétation de cette violence, il semble utile de partir d'un questionnement balançant entre deux pôles : violences anomiques et violences expressives. Le premier item désigne des formes de violences suscitées par la désorganisation de l'espace social et l'affaiblissement des opportunités qu'il offre à la jeunesse, les manifestations violentes étant alors le médium via lequel s'exprime le "sentiment de rage " face à l'impasse sociale et identitaire, et à l'exclusion ressentie. ${ }^{(37)}$ Le deuxième pôle, qui ne fonctionne pas à l'exclusion du premier, signale au contraire la dimension expressive, voire revendicative - et finalement politique - des violences juvéniles, témoignant d'une
1940 ", Journal of Social History, 37, 4, Summer 2004, p. 829-851.

(34) Andrew Davies, "These Viragoes are no less Cruel than the Lads: Young Women, Gangs and Violence in Late Victorian Manchester and Salford ", British Journal of Criminology, 39, 1, 1999 , p. 72-89.

(35) Elsa Dorlin, La matrice de la race. Généalogie sexuelle et coloniale de la nation française, Paris, La découverte, 2006, 307 p. ; Nicole Edelman, Les métamorphoses de l'hystérique, du début du XIXème siècle à la Grande Guerre, Paris, La découverte, 2003, p. 89, et le chap. 6 :

"Les paroxysmes de l'hystérie : violence et folie (années-1870-1880)", p. 110-146.

(36) Margo De Koster, Weerbaar, weerspannig of crimineel? Meisjes en jonge 
vrouwen tussen emancipatie

en delinquentie tijdens de eerste helft van de twintigste

eeuw [Résistantes, récal-

citrantes ou criminelles?

Filles et jeunes femmes

entre émancipation

et délinquance durant la

première moitié du

XXème siècle], thèse de

doctorat en histoire, Vrije

Universiteit Brussel,

2003 ; Pamela Cox,

Gender, Justice and

Welfare : Bad Girls in

Britain, I900-I950,

London, Palgrave

MacMillan, 2003, 272 p. ;

Anne Meis Knupfer,

Reform and Resistance.

Gender, Delinquency, and

America's First Juvenile

Court, New York

Routledge, 2001, 290 p. ;

Tamara Myers, Caught :

Montreal's Modern

Girls and the Law, I860-

I945, Toronto, University

of Toronto Press,

2006, 345 p.
(37) Cette question

traverse la pensée sociologique, de Durkheim

à Dubet, en passant par

Merton.

(38) Axel Honneth, La

lutte pour la

reconnaissance, Paris, Cerf,

2000, 232 p. ; Gérard

Mauger, Les bandes,

le milieu et la bohème

populaire. Etudes de socio-

logie de la déviance des

jeunes des classes populaires

(1975-2005), Paris, Belin,

2006, 252 p.

(39) Cf. Thomas

Sauvadet : Le capital

guerrier. Concurrence et

solidarité entre jeunes

de cité, Paris, Armand

Colin, 2006 ; « Le senti-

ment d'insécurité du

“dealer de cité” ", Sociétés

et jeunesses en difficulté,

$\mathrm{n}^{\circ} 1$, mars 2006,

http://sejed.revues.org/

(40) Robert Castel,

« La discrimination négative : le déficit de citoyenneté des jeunes de ban- aspiration à la reconnaissance. ${ }^{(38)}$ Lintrication de ces deux registres d'action apparemment contradictoires est finement soulevée par Ivan Jablonka. Ainsi, la violence des jeunes de l'Assistance sur les animaux de la ferme relève a priori du geste désespéré, gratuit, purement malveillant. Mais à y regarder de près, au vu de l'importance économique, voire affective que revêtent ces bêtes dans l'économie matérielle et sensible des campagnes du premier XXème siècle, leur châtiment relève bien aussi d'un geste stratégique, spécialement lorsqu'il est porté par des jeunes dont la valeur économique décroît avec les restrictions du travail juvénile au profit de leur scolarisation.

Enfin, il faut aussi s'interroger sur la véritable nature déviante de la violence. Cette violence n'est pas " gratuite » et ne relève pas d'un phénomène désorganisé : dans bien des cas, son emploi est strictement régulé par le code de conduite du groupe qui en fait usage, lequel s'inscrit dans un ensemble de pratiques sociales, comme le montrent plusieurs auteurs de ce volume. La violence recèle ici, paradoxalement, une dimension conformiste, permettant au groupe, à l'instar de la "puissance " publique, d'imposer des formes de régulations socialement reconnues et acceptées. Dans les sociétés pré-étatiques, les groupes de jeunesse occupent ce rôle régulateur, avec le charivari. Aude Musin et Élise Mertens indiquent bien que les jeunes ne semblaient pas, aux XIVème et XVème siècles, plus fréquemment poursuivis par la justice pour des faits de violence, ce qui laisse augurer une grande tolérance à l'égard de son exercice. François Ploux et Aurélien Lignereux, quant à eux, perçoivent une transition dans la France rurale du premier XIXème siècle. Si l'assignation sociale de la jeunesse à certaines formes de violences codifiées reste étonnement prégnante, la justice, bras armé de l'État, tente alors, avec diffi- 
culté, de s’immiscer dans ces querelles intercommunales cycliques et d'une rudesse extrême.

Aujourd'hui, retour des choses, c'est bien souvent là où l'État faillit dans son rôle de régulateur que s'impose de nouveau la nécessité du recours à la violence. Il s'agit, d'une part, de régler un marché économique alternatif (trafics) qui, bien qu'illicite, répond aux grands canons du libéralisme et déploie ainsi une violence systémique inouïe, ${ }^{(39)}$ mais aussi, d'autre part, de solliciter les pouvoirs publics face à une série de discriminations spatiales et raciales vécues comme un abandon social. ${ }^{(40)}$ Mais il ne s'agit pas simplement ici d'une réponse au retrait de l'État de ses prérogatives sociales. Car les jeunes des cités, loin de n'être que les nouvelles victimes du dérèglement des sociétés " providencialistes ", reprennent sans doute là le flambeau de la lutte sociale et identitaire à laquelle avaient dû renoncer leurs aînés face aux violences d'État, qu'elles soient " massacres » ou discriminations insidieuses. ${ }^{(41)}$

Au terme de ce survol, on perçoit bien que la violence n'est pas le fait d'une catégorie sociale spécifique, qu'elle soit définie par l'âge, le sexe, la classe, ou l'origine ethnique. Si elle témoigne de véritables rapports de force entre groupes sociaux, elle relève également de procédés arbitraires de désignation qui occultent leur genèse complexe. Redondante, la violence se redouble puisque l'énoncé même de son assignation à certains groupes relève en soi de la violence symbolique, comme en témoigne la prégnance contemporaine de l'association de la jeunesse à la violence. Cet amalgame relève autant des troubles causés par une partie de la jeunesse que de la crise de l'action politique. lieue ", Annales HSS,

$\mathrm{n}^{\circ} 4$, juillet-août 2006,

p 777-808.

Il est inquiétant de

constater qu'aucun

historien n'est intervenu

dans ce numéro des

Annales, et plus largement dans l'entreprise nécessaire de décryptage des révoltes de novembre 2005.

(41) On attendrait beaucoup de postcolonial studies " à la française ", qui nous éclaireraient sur la répression étatique des expressions identitaires depuis les années 1950. Alain Dewerpe, Charonne, 8 février 1962 : anthropologie historique d'un massacre d'État, Paris, Gallimard, 2006, 897 p. ;

Abdelmalek Sayad, La double absence : des illusions de l'émigré aux souffrances de l'immigré, Paris, Le Seuil, 1999, 437 p. ; Michel Kokoreff, La force des quartiers. De la délinquance à l'engagement politique, Paris, Payot, 2003, 349 p. 